\title{
Robust Nanogenerator Based on Vertically Aligned ZnO Nanorods Using Copper Substrate
}

\author{
Naveed Sheikh, ${ }^{1}$ Nitin Afzulpurkar, ${ }^{1}$ and Muhammad Waseem Ashraf ${ }^{2}$ \\ ${ }^{1}$ School of Engineering and Technology, Asian Institute of Technology (AIT), Pathumthani, Bangkok 12120, Thailand \\ ${ }^{2}$ Department of Physics (Electronics), GC University, Lahore 54000, Pakistan
}

Correspondence should be addressed to Naveed Sheikh; naveedaitbangkok@gmail.com

Received 19 April 2013; Accepted 29 July 2013

Academic Editor: Yangchuan Xing

Copyright (c) 2013 Naveed Sheikh et al. This is an open access article distributed under the Creative Commons Attribution License, which permits unrestricted use, distribution, and reproduction in any medium, provided the original work is properly cited.

\begin{abstract}
We report the growth of well-aligned $\mathrm{ZnO}$ nanorods that were successfully synthesized on copper $(\mathrm{Cu})$ substrate by using a simple hydrothermal method at low temperature leading to a robust large area nanogenerator (NG) based on cost effective Cu electrode which could enable energy harvest from the walking motions. First, the seed layer of gold (Au) has been deposited by plasma sputtering. Then, the growth process of nanorods was carried out in a sealed chemical bath. The lengths of nanorods 5-6 $\mu \mathrm{m}$ were achieved. The longer and bigger nanorods produced a surface with larger contact area and higher roughness. The larger contact area improves the absorption rate of incident light and the rougher surface strengthens the scattering effect. The surfaces were characterized by X-ray diffraction (XRD), scanning electron microscope (SEM), and energy dispersive X-ray spectroscopy (EDX). Then, $\mathrm{Cu}$ substrate was used for the development of high-output NG. Twenty-seven NGs were developed with maximum output voltage that exceeded to $1.8 \mathrm{~V}$ and maximum output current that exceeded to $148 \mathrm{nA}$. The structural simulation of nanorod has also been performed in ANSYS. The Cu substrate-based NG provides a feasible technique for effectively converting mechanical energies to electrical energy from external force.
\end{abstract}

\section{Introduction}

Nano structured materials have received extensive attention from researchers in the last few years because of their novel properties which differ from those of bulk materials $[1,2]$. Nanocrystalline materials exhibit completely different properties due to their enhanced surface to volume ratio and quantum confinement effects. Zinc oxide $(\mathrm{ZnO})$ is one of the important materials and photonic material that attracts much attention for nanodevice applications such as nanogenerators, biomedical devices, sensors, transducers, and optoelectronics because of its direct bandgap of $(3.37 \mathrm{eV})$ and large exciton binding energy $(60 \mathrm{meV})$ at room temperature. $\mathrm{ZnO}$ can be made highly conductive by doping and transparent to visible light. Several reports on the characterization of unidimensional nanorods of compound and elemental semiconductors such as GaAs [3], Ge [4], Si [5], and $\mathrm{ZnO}$ [6-8] are available in the literature. Currently, there is an increasing interest in the fabrication of $\mathrm{ZnO}$ with the various morphology and uniform size, including low-dimensional $\mathrm{ZnO}$ nanostructures such as nanowires, nanosheets, nanoflowers, nanotubes, nanorods, nanocombs, nanorings, nanobelts, nanohelices, nanobows, and nanocages, which have been successfully synthesized by different approaches such as thermal evaporation method, microwave heating, nonaqueous approaches, chemical vapor deposition (CVD), sol-gel process, hydrothermal process, gas condensation, laser ablation, template-assisted growth, and refluxing method $[9,10]$. One of the most inexpensive and energy-efficient strategies for synthesizing $\mathrm{ZnO}$ nanorods is the hydrothermal process which does not require complex vacuum environment or high temperature. The hydrothermal process induces anisotropic crystal growth in a solution [11, 12]. The hydrothermal process provides good control over the morphology of the nanorods grown [13]. The growths of $\mathrm{ZnO}$ nanorods on the flat substrates such as Si $[13,14]$ glass [15], and carbon cloth $[16,17]$ have been reported. However, there have been no reports of $\mathrm{ZnO}$ nanorods grown on Copper $(\mathrm{Cu})$ substrate. The $\mathrm{ZnO}$ crystal is hexagonal wurtzite with lattice parameters $a=0.3296 \mathrm{~nm}$ and $c=0.52065 \mathrm{~nm}$. 
The $\mathrm{ZnO}$ structure can be described as series of alternating planes composed of tetrahedrally coordinated $\left(\mathrm{O}^{2-}\right)$ and $\left(\mathrm{Zn}^{2+}\right)$ arranged along the $c$-axis. The basal plane (001) is the most common polar surface. One end of the basal polar plane terminates in partially negative oxygen lattice points and the other end is terminated in partially positive $\mathrm{Zn}$ lattice points.

The idea of human motion-based energy scavenging has enormous technological interest in accordance with the emergent potential of portable smart electronics [18]. It is becoming more realistic, due to advanced research in nanoelectronics, to run these devices at tremendously low power consumption, so that energy harvested from the environment may be adequate to meet the operational mode energy needs [19]. Thermal and solar energy are the most common and practical sources of energy to be harvested from our surroundings. However, these forms of energy are time and local dependent. With the advances in technology developed for small-scale energy harvesting, it may be possible to scale up this type of technology at a large scope such as with ocean waves and wind power. Such potential needs to be explored with the consideration of the scalability of the technology, the cost of materials and fabrication process, environmental impact, and practical feasibility for implementation [20]. Mechanical energy is one of the most representative sources that can be artificially generated from vibration, movement of automobile, and human walking, all of which are usually wasted [21]. The fundamental mechanism of a nanogenerator (NG) is related to a piezoelectric potential generated in nanorods when they are dynamically strained under an external force, and the corresponding transient current that flows to balance the Fermi levels at two contacts $[22,23]$. The NG is still difficult to apply to energy harvesting device to harvest the mechanical energy from the environment owing to weak durability, high cost, and low-throughout process. Thus, it is necessary to develop strategies towards achieving robust and cost-effective NG in order to consistently scavenge the mechanical energy from the environmental sources.

Huang et al. [24] reported $\mathrm{ZnO}$ nanorod arrays thin film bilayer structure for homojunction diode and highperformance memristor to complementary 1D1R application. Faisal et al. presented synthesis, characterizations, photocatalytic, and sensing studies of $\mathrm{ZnO}$ nanocapsules [25]. Faisal et al. reported fabrication of $\mathrm{ZnO}$ nanoparticles based on sensitive methanol sensor and efficient photocatalyst [26]. Ding et al. presented the growing of vertical $\mathrm{ZnO}$ nanorod arrays within graphite [27]. Wrobel et al. reported direct synthesis of $\mathrm{ZnO}$ nanorod field emitters on metal electrodes [28]. Liu et al. studied fabrication of $\mathrm{ZnO} / \mathrm{CdS} / \mathrm{Cu}_{2} \mathrm{ZnSnS}_{4} \mathrm{p}$-n heterostructure nanorod arrays via a solution-based route [29]. Chen et al. reported synthesis of grapheme $\mathrm{ZnO}$ nanorod nanocomposites with improved photoactivity and antiphotocorrosion [30]. Umar et al. reported ultrasensitive cholesterol biosensor based on low-temperature grown $\mathrm{ZnO}$ nanoparticles [31]. Hu et al. presented a portable flow-through fluorescent immunoassay labon-a-chip device using $\mathrm{ZnO}$ nanoroddecorated glass capillaries [32]. Gao et al. presented nanorodnanosheet hierarchically structured $\mathrm{ZnO}$ crystals on zinc foil as flexible photoanodes for dye-sensitized solar cells [33]. Jiang et al. [34] reported carrier concentration-dependent electron transfer in $\mathrm{Cu}_{2} \mathrm{O} / \mathrm{ZnO}$ nanorod arrays and their photocatalytic performance. Sheikh et al. reported pyramid shape $\mathrm{ZnO}$ solid microstructures for energy harvesting, drug delivery, and skin treatment [35].

In general, since a lightweight and cost-effective $\mathrm{Cu}$ is good electrical conductor, it has been extensively used in different industries. In order to prevent the detachment of $\mathrm{ZnO}$ nanorods from the substrate at the boundary interface under an applied strain, a microscale rough surface of the $\mathrm{Cu}$ substrate was produced to increased the surface contact area by rubbing with sandpaper prior to growth of $\mathrm{ZnO}$ nanorods. However, rubbing with sandpaper $\mathrm{Cu}$ substrate surface exhibits enhanced hydrophilicity due to increased roughness. For this reason, $\mathrm{ZnO}$ nanorods grow uniformly on the large area. Once $\mathrm{ZnO}$ nanorods are grown on the one side of $\mathrm{Cu}$ substrate, the other side can be used as upper electrode of lower unit. The working principle of the nanogenerator is related to the coupling of piezoelectric and semiconducting properties. When a stress is applied by an external force, the $\mathrm{ZnO}$ nanorods grown parallel to the $c$-axis are under uniaxial compression. Negative and positive piezoelectric potential, respectively, occur at the top and bottom side of the $\mathrm{ZnO}$ nanorods, the corresponding transient current flows from the tip and bottom through the external circuit, which is detected as an electric pulse. As the compressive strain is released by the removal of the external force, the piezoelectric potential in the nanorods disappeared. As a result, the electrons flow back via the external circuit, creating an electrical pulse in opposite direction. These electrical pulses can be accumulated, and the amount can be significantly enhanced by integration in parallel and series.

In this work, we have used hydrothermal method to synthesize $\mathrm{ZnO}$ nanorods on $\mathrm{Cu}$ substrate leading to a robust large-area NG based on cost effective $\mathrm{Cu}$ electrode which could enable energy harvest from the walking motions. copper-based energy storage systems have tremendous advantages including high specific energy, low cost and zero emissions. What makes this work very interesting is the fact that the $\mathrm{ZnO}$ nanorods grown on a Cu substrate can be used to make a NG which is low-cost approach to converting mechanical vibration into electricity.

\section{Simulation}

The NG has been developed by using $\mathrm{ZnO}$ nanorods. The electrical energy has been achieved by applying the force or mechanical movement on the surface of nanorods. When the force is applied on the top surface of NG, then, the deposited nanorods exhibit piezoelectric behavior. This behavior has been studied in finite element software ANSYS by considering single nanorod. First, the 3D model has been built in ANSYS parametric design language. Then, material properties of $\mathrm{ZnO}$ have been defined and brick meshing was performed by using element 20 nod186. Then initial condition has been setup. The force of 0.1 to $1 \mathrm{~N}$ has been applied on single nanorod and then solution has been achieved. The deflection 


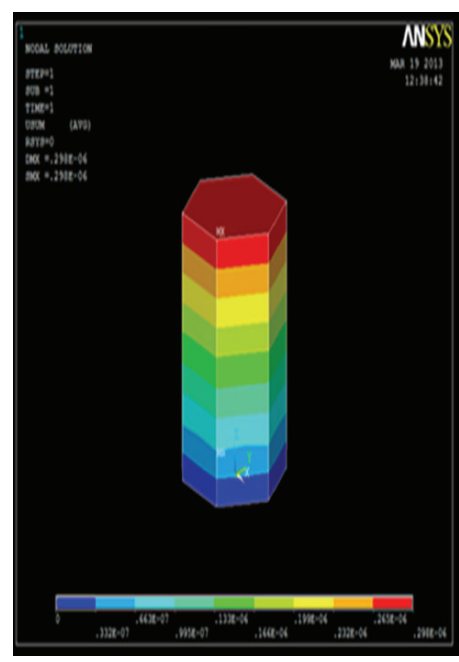

(a)

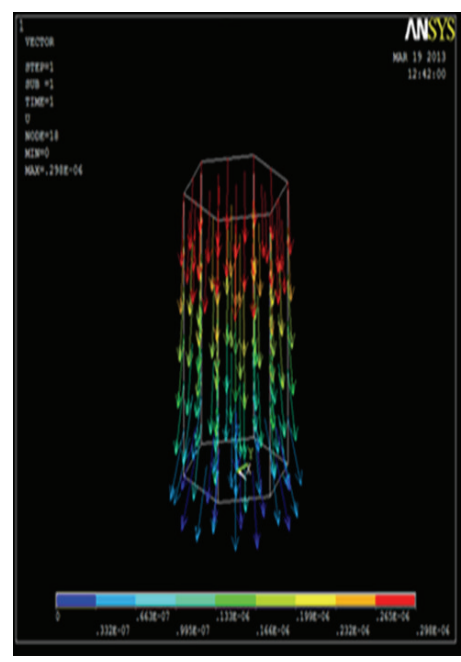

(b)

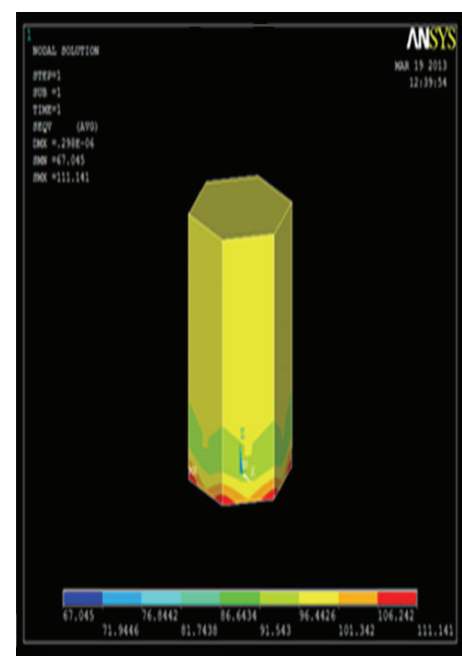

(c)

Figure 1: (a) Deflection, (b) deflection in vector form, and (c) stress distribution.

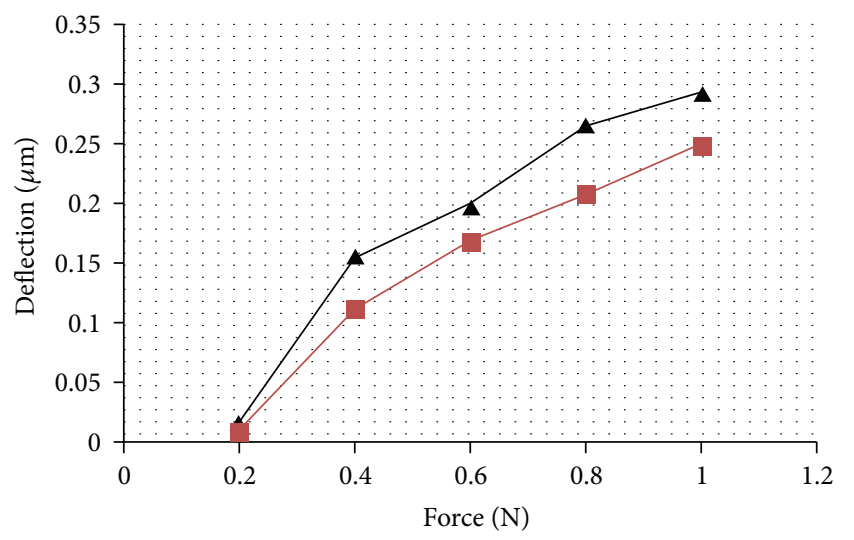

$\longrightarrow$ Simulated

$\rightarrow-$ Experimental

FIGURE 2: Deflection at different applied forces.

and stress on nanostructure has been analyzed. It has been observed that the stress values are well below the yield strength of material. The simulation results of nanorods in ANSYS parametric design language are shown in Figure 1.

It has been observed that the maximum deflection $0.294 \mu \mathrm{m}$ has been achieved by applying the force of $1 \mathrm{~N}$. The deflection of nanorods has also been calculated experimentally by using laser displacement measurement method. The simulated and experimental results are in close agreement and shown in Figure 2.

\section{Experimental Section}

3.1. Deposition of $\mathrm{Cr} / \mathrm{Au}$ Seed Layer on Copper (Cu) Substrate via Plasma Sputtering. To prepare the $\mathrm{Cr} / \mathrm{Au}$ seed layer, all the regents were analytically uncontaminated and used without further purification. The substrate was cleaned in ultrasonic bath with water, ethanol, and acetone for 15 minutes. The treated substrate was then impregnated ultrasonically in distilled water and dried in an oven at $200^{\circ} \mathrm{C}$ for 1 hour. After that the substrate was again treated with $1 \%$ dodecane thiol solution in ethanol for 2 hours and then heated at $120^{\circ} \mathrm{C}$ for 15 minutes prior to $\mathrm{ZnO}$ nanorods grown. Then, $20 \mathrm{~nm}$ layer of chromium (Cr) and $50 \mathrm{~nm}$ dense layer of $\mathrm{Au}$ were successively deposited on top of the $\mathrm{Cu}$ substrate by plasma sputtering. The chromium thin layer served as an adhesion layer and the $\mathrm{Au}$ dense film was expected as an intermediate layer to assist growth. The $\mathrm{Cu}$ substrate was then annealed at $160^{\circ} \mathrm{C}$ for 1 hour to increase the crystallininty of the gold dense film.

\subsection{Synthesis of $\mathrm{ZnO}$ Nanorods via Hydrothermal Method.} The Au seeded substrate was suspended upside down in sealed chemical bath containing equimolar solution $(10 \mathrm{mM})$ of zinc nitrate hexahydrate $\left(\mathrm{Zn}\left(\mathrm{NO}_{3}\right)_{2} \cdot 6 \mathrm{H}_{2} \mathrm{O}\right)$ and hexamethylenetetramine $\left(\mathrm{C}_{6} \mathrm{H}_{12} \mathrm{~N}_{4}\right)$ in deionized (DI) water and was then positioned in a hot plate stirrer and heated to $95^{\circ} \mathrm{C}$ for $5 \mathrm{~h}$. Different concentrations of precursor solution were used to study the growth of $\mathrm{ZnO}$ nanorods. The precursor solution was reformed every $5 \mathrm{~h}$ and growth was continued up to 20 hours. Throughout this process, bubbles produced in the solution rise to the solution surface and are trapped at the face-down copper substrate surface, preventing uniform growth of $\mathrm{ZnO}$ nanorods on the large-area surface. However, after rubbing with the sand paper the $\mathrm{Cu}$ substrate exhibits improved hydrophilicity due to enhanced roughness as well as the surface oxidation because of the rubbing process. Consequently, the bubbles do not adhere to the surface as the molecules of the solution are strongly attached to the hydrophilic surface. For this reason, $\mathrm{ZnO}$ nanorods grow uniformly on the $\mathrm{Cu}$ substrate. The samples were then heated at $150^{\circ} \mathrm{C}$ for 30 minutes to vaporize any organic deposits. The substrate was rinsed with DI water to remove any residual salt from the surface and dried in air. In order to process the model for NG device, we spun coated polymethylmethacrylate (PMMA) film, to electrically separate adjacent nanorods 


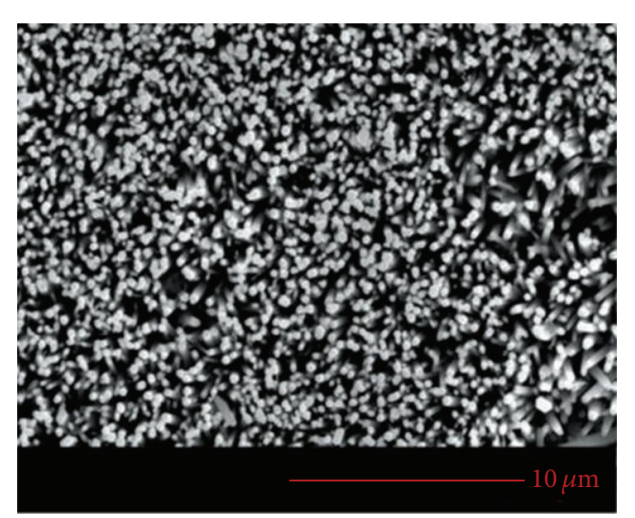

(a)

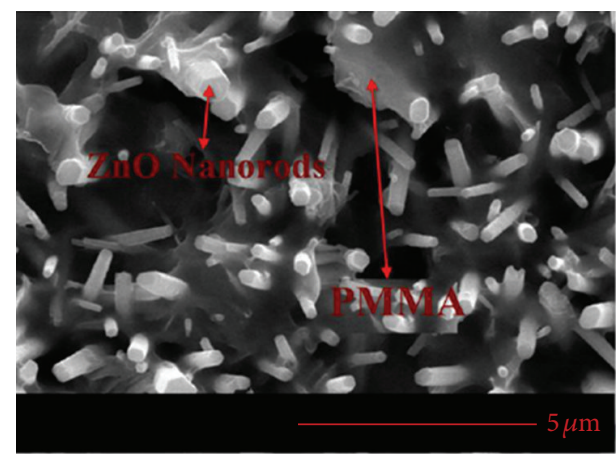

(c)

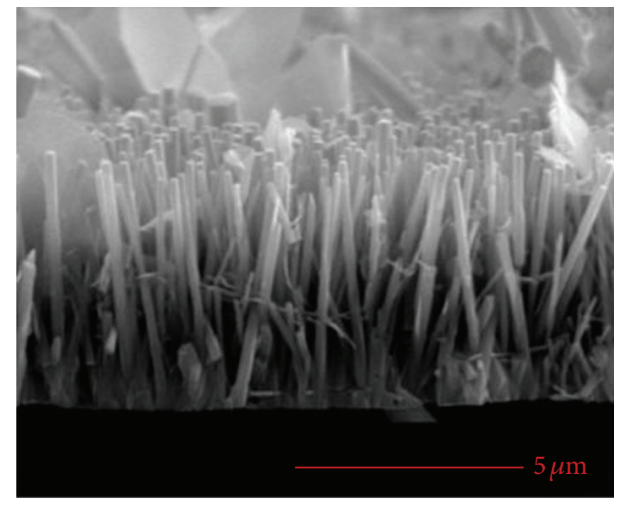

(b)

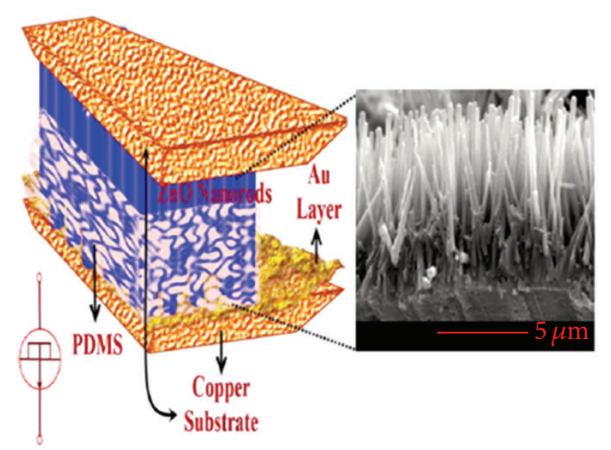

(d)

Figure 3: (a) Top view of $\mathrm{ZnO}$ nanorods on copper substrate, (b) cross-sectional view of $\mathrm{ZnO}$ nanorods on copper substrate, (c) ZnO nanorods with insulating layer after oxygen plasma etching, and (d) schematic illustration of the individual nanogenerator and its symbol.

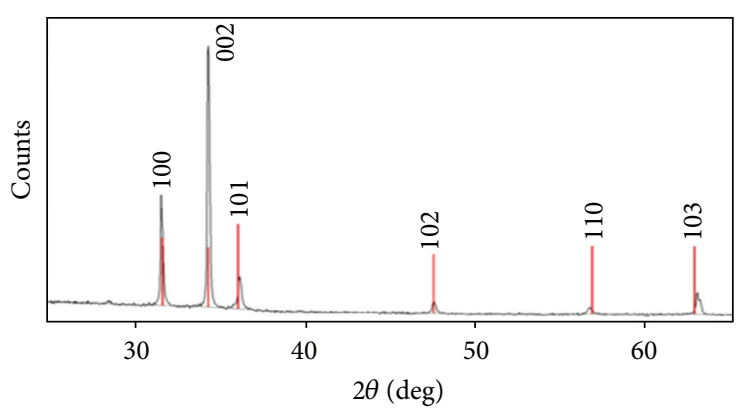

(a)

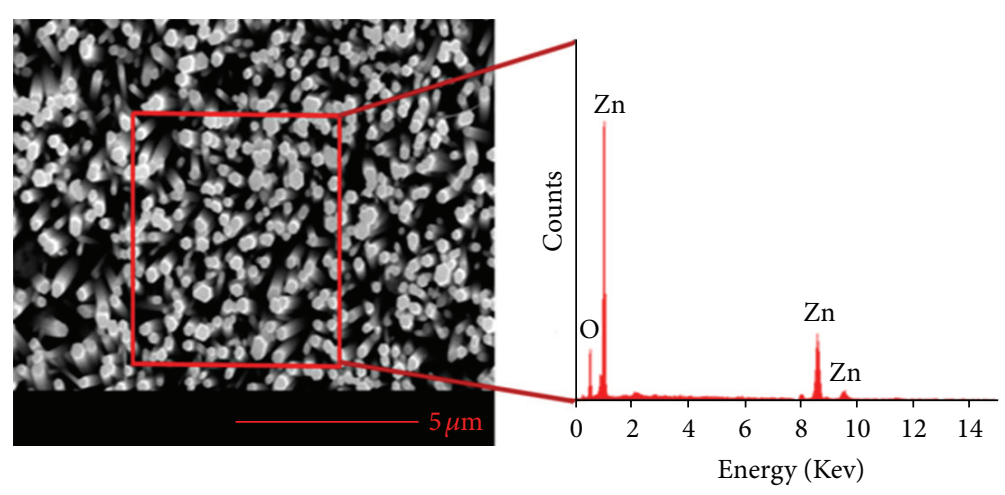

(b)

Figure 4: (a) XRD pattern of $\mathrm{ZnO}$ nanorods grown on $\mathrm{Cu}$ substrate and (b) EDX spectrum of prepared ZnO nanorods. 


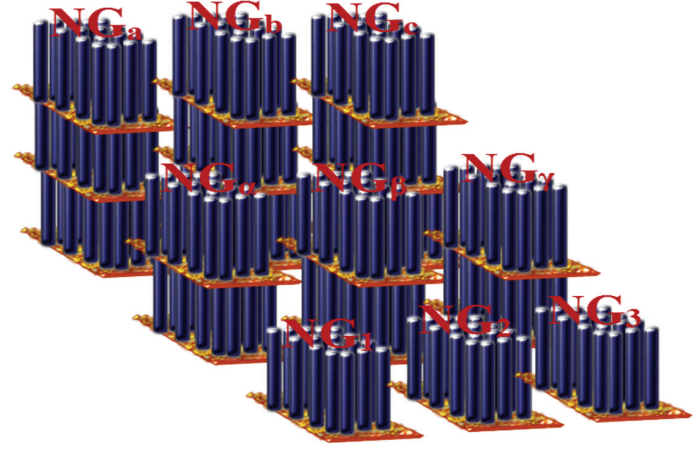

FIGURE 5: Schematic illustration of integrated NG.

from each other, at a speed of $2000 \mathrm{rpm}$ for 30 seconds and then annealed it at $100^{\circ} \mathrm{C}$ for 1 minute. Reactive ion etching was used to etch PMMA layer to expose the tips of the nanorods. The results of fabrication will be given in the next section.

\section{Results and Discussion}

4.1. Characterization. The characterization was performed to determine morphology and size of the $\mathrm{ZnO}$ nanorods by using SEM (Hitachi S3400, operated at $20 \mathrm{kV}$ ). The crystalline structure of aligned $\mathrm{ZnO}$ nanorods was observed using XRD (Bruker, D8 Advance). The chemical composition was characterized by using energy dispersive spectroscopy (EDX). Then, NG were assembled and tested. The, SEM image of grown nanorods on $\mathrm{Cu}$ substrate is shown in Figure 3.

SEM image of spin coated PMMA layer is shown in Figure 3(c). The image undoubtedly indicates that spin coating method of the insulating layer seals the gap between the nanorods and behaves like a protecting shield to insulate them from each other. The surface of the nanorods wrapped with PMMA is etched away after exposing to oxygen in the reactive ion etching. The zinc oxide nanorods were hexagonal and vertically aligned on the copper substrate. The diameter and length of the $\mathrm{ZnO}$ nanorods were $350-400 \mathrm{~nm}$ and 5$6 \mu \mathrm{m}$, respectively. SEM images reveal that the nanorods array with the largest length and diameter was synthesized by the hydrothermal method.

The crystalline structure of aligned $\mathrm{ZnO}$ nanorods was observed using XRD with $\mathrm{CuK} \alpha$ radiation, $\lambda=0.154 \mathrm{~nm}$ as shown in Figure 4(a) and EDX spectrum of the $\mathrm{ZnO}$ nanorods as shown in Figure 4(b). It is clear from Figure 4(b) that atomic ratio of $\mathrm{Zn}$ and oxygen $(\mathrm{O})$ is ca. 1:1 that is indicating the perfect stoichiometric chemical composition of the $\mathrm{Zn}$ and $\mathrm{O}$ in the nanorods.

XRD pattern of $\mathrm{ZnO}$ nanorods illustrates 6 reflection peaks of hexagonal wurtzite structure. These are (100), (002), (101), (102), (110), and (103) at $2 \theta$ with corresponding angle $31.8^{\circ}, 34.4^{\circ}, 36.2^{\circ}, 47.6^{\circ}, 56.6^{\circ}$, and $63.0^{\circ}$, respectively. Compared with the other peaks, the (002) diffraction peak is much stronger in intensity. That confirms the nanorods were aligned in the $c$-axis. Hence, they are acceptable in accordance with (JCPDS) card no. 36-1451 with lattice constant $a=3.24 \AA$ and $c=5.21 \AA$. The strong intensity and narrow full width at half maximum (FWHM) of the XRD pattern shows that the $\mathrm{ZnO}$ nanorods synthesized had a good crystalline structure. To confirm the composition of the grown nanorods, EDX analysis was performed. It is confirmed from the EDX analysis that the grown nanorods are indeed composed of $\mathrm{Zn}$ and $\mathrm{O}$ only. The molecular ratio of $\mathrm{Zn}$ : $\mathrm{O}$ of the grown nanorods is evaluated from EDX and quantitative analysis data is close to $1: 1$. No other peak for any other element has been found in the spectrum except $\mathrm{O}$ and $\mathrm{Zn}$ which again confirmed that the grown nanorods are pure $\mathrm{ZnO}$.

4.2. Scheme for the Integration of (NG) and Characterization. The nanogenerator based on $\mathrm{Cu}$ substrate can easily be integrated in parallel and series for increasing output voltage and current owing to the uniform growth of $\mathrm{ZnO}$ nanorods on $\mathrm{Cu}$ substrate and the simultaneous use of $\mathrm{Cu}$ substrate as lower and upper electrodes as shown in Figure 5.

To characterize the electrical performance of the nanogenerator we integrated the NG in series and parallel combination, consisting of nine parts on the large area of $\mathrm{Cu}$ electrode. The first combination including NG1, NG2, and NG3 was incorporated in parallel with three parts forming one unit. The second combination including $\mathrm{NG} \alpha, \mathrm{NG} \beta$, and $\mathrm{NG} \gamma$, was incorporated in parallel with three parts attached in series with two units. The third combination including $\mathrm{NGa}$, NGb, and NGc, was integrated in parallel with three parts serially attached with three units. To characterize the performance of the NG, the NG array was tested by a convenient trigger system which could periodically compress and release at frequency of $3-4 \mathrm{~Hz}$. In case of serial combination, we calculated the output voltage with the increase in the number of serially incorporated unit. As the number of incorporated units increases from one to three in the order of $\mathrm{NG1}, \mathrm{NG} \alpha$, and $\mathrm{NGa}$ and their output voltage increases from $0.03 \mathrm{~V}, 0.09 \mathrm{~V}$, and $0.13 \mathrm{~V}$, respectively. The units integrated in series are simultaneously affected by the same external force and their corresponding piezoelectric potential in external load is summed, the output voltage increases with the number of units, the output voltage also increases in the order of NG2, NG $\beta$, and NGb with $0.04 \mathrm{~V}$, $0.09 \mathrm{~V}$, and $0.18 \mathrm{~V}$, respectively. The possibility of serial integration based on copper $\mathrm{Cu}$ in order of $\mathrm{NG} 3, \mathrm{NG} \gamma$, and $\mathrm{NGc}$ with output voltage increases as $0.03 \mathrm{~V}, 0.07 \mathrm{~V}$, and $0.17 \mathrm{~V}$, respectively. The all above combination is shown in Figure 6.

After that, we measured the output current for the NG integrated in parallel with three parts, the parallel integration of three parts including NG1, NG2, and NG3 led to the output current of $18 \mathrm{nA}$. The 2 nd integration which was composed of two layers including $\mathrm{NG} \alpha, \mathrm{NG} \beta$, and $\mathrm{NG} \gamma$ led to the output current of $38 \mathrm{nA}$. The 3 rd integration which was composed of three layers including $\mathrm{NGa}, \mathrm{NGb}$ and $\mathrm{NGc}$ led to the output current of $97 \mathrm{nA}$. The $\mathrm{NG}$ based on $\mathrm{Cu}$ substrate can be simply integrated not only in series for high output but also in parallel for the high output current without any specific size limit. Since the human walking and movement of automobiles are usually occurring at various low frequencies, 

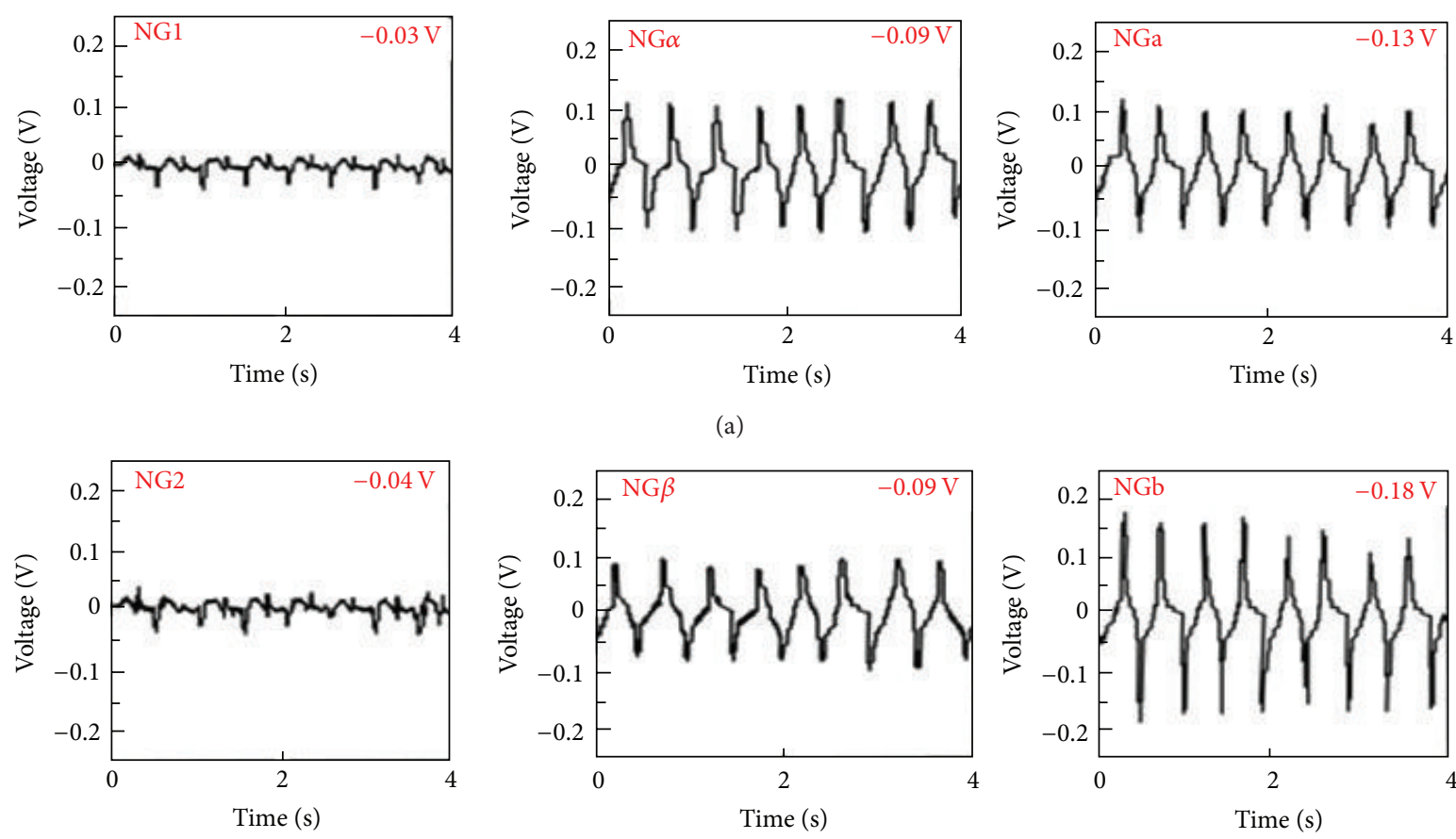

(a)
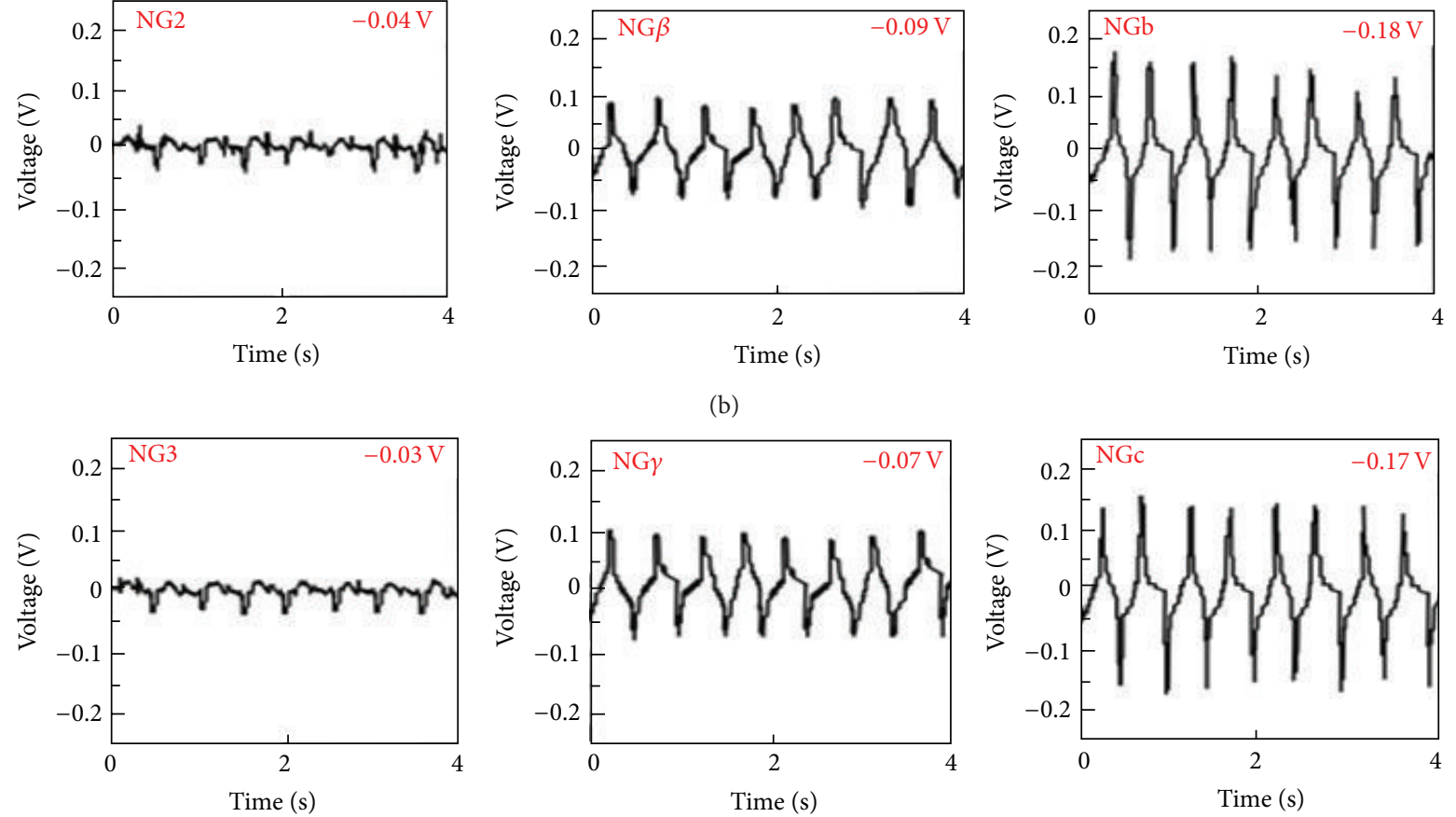

(b)
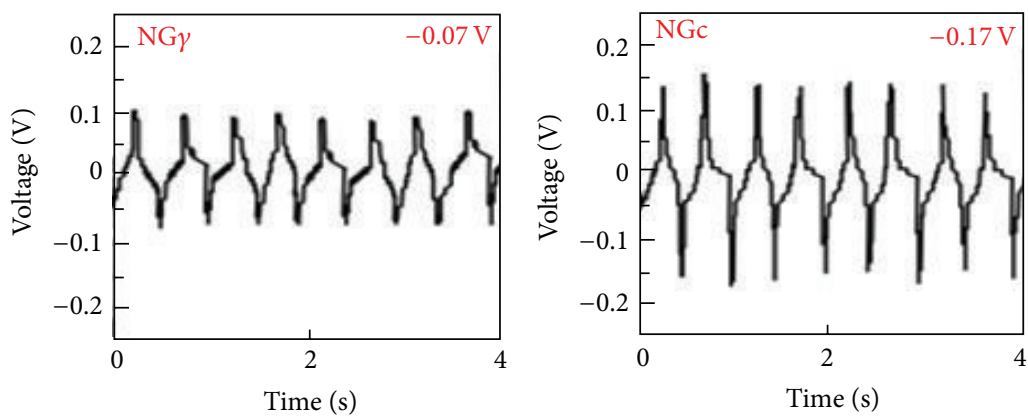

(c)

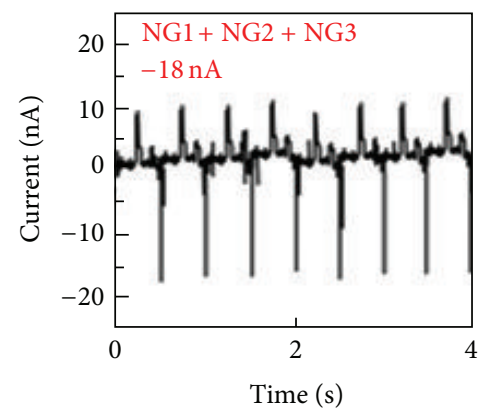

(d)

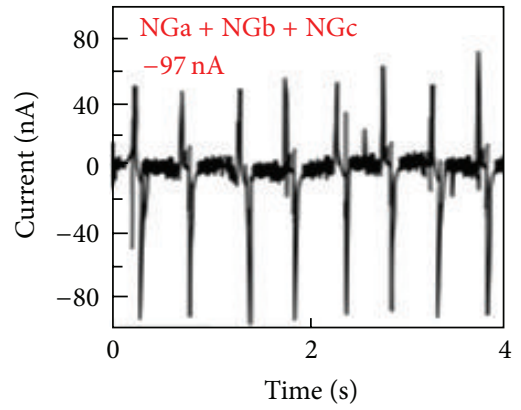

(e)

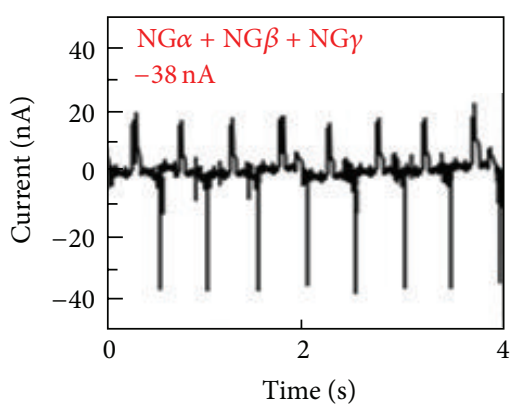

(f)

FIGURE 6: (a) Incorporated units in the order of NG1, NG $\alpha$, and $\mathrm{NGa}$ and their output voltages $0.03 \mathrm{~V}, 0.09 \mathrm{~V}$, and $0.13 \mathrm{~V}$; (b) incorporated units in the order of NG2, NG $\beta$, and NGb and their output voltages $0.04 \mathrm{~V}, 0.09 \mathrm{~V}$, and $0.18 \mathrm{~V}$; (c) incorporated units in the order of NG3, $\mathrm{NG} \gamma$, and NGc and their output voltages $0.03 \mathrm{~V}, 0.07 \mathrm{~V}$, and $0.17 \mathrm{~V}$; (d) output current of $18 \mathrm{nA}$ by parallel combination of NG1, NG2, and NG3; (e) output current of $97 \mathrm{nA}$ by three layered including NGa, NGb, and NGc; (f) output current of $38 \mathrm{nA}$ by two layered combination including $\mathrm{NG} \alpha, \mathrm{NG} \beta$, and $\mathrm{NG} \gamma$.

these stable results are promising for the use in practical applications. Photograph image of real device and schematic illustration of three-dimensional integrated nano device is shown in Figure 7.
Here, we design the device for energy scavenging from human walking that is composed of three units. The device was tightly fixed on smooth supporting to prevent the electrical noise. Under the human walking condition, 


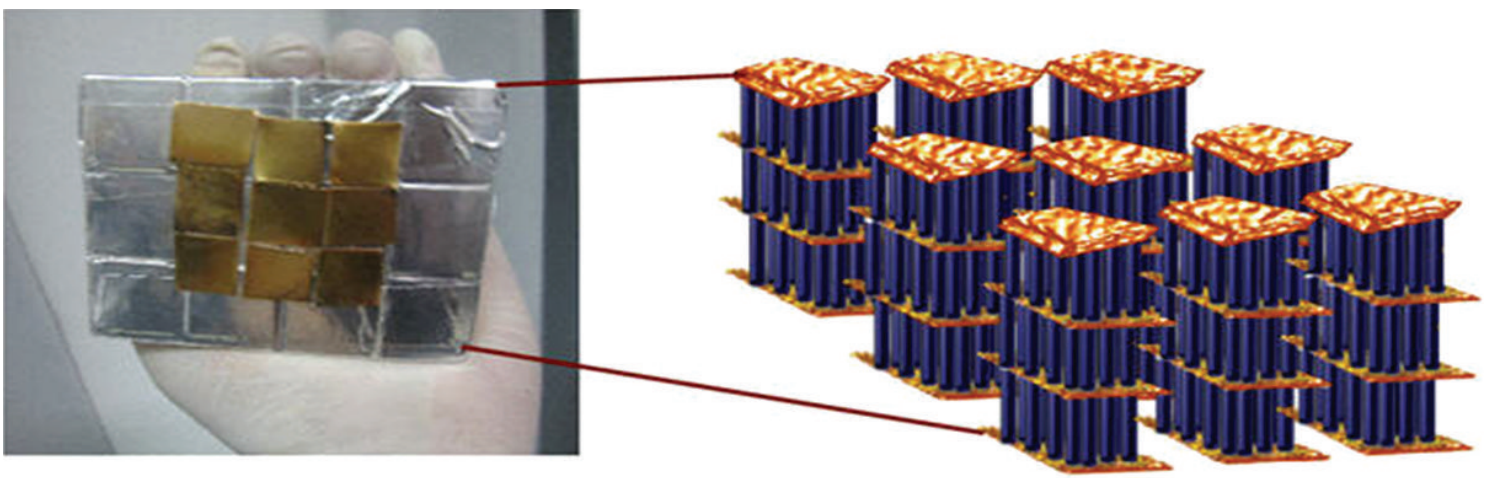

(a)

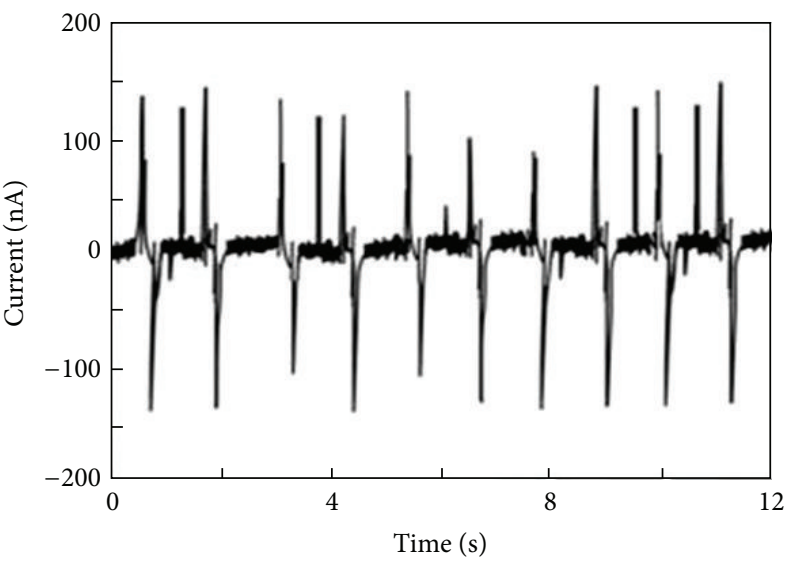

(b)

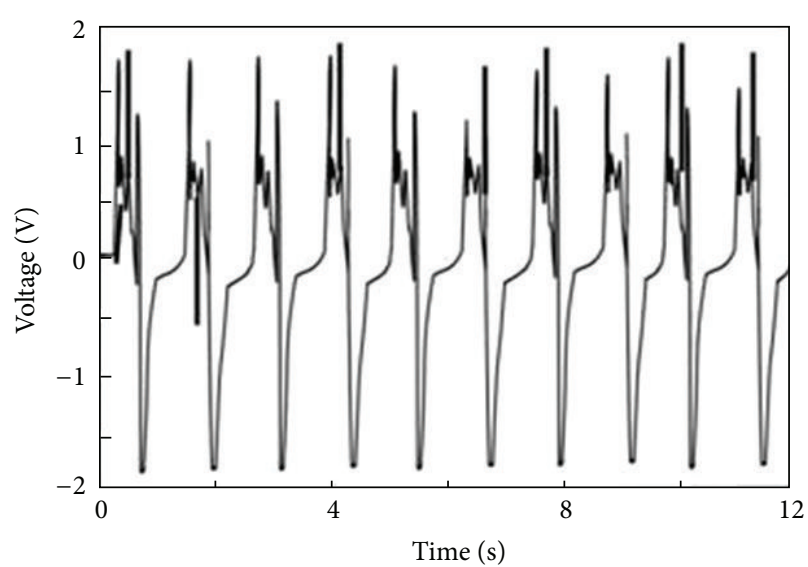

(c)

FIGURE 7: (a) Image of real device and schematic illustration of three dimensional integrated nanodevice. (b) Maximum output voltage of the nanodevice. (c) Maximum output current of the nanodevice.

the nano device exhibited maximum output current up to $148 \mathrm{nA}$ and maximum output voltage up to $1.8 \mathrm{~V}$. The output voltage and current are totally dependent on the force acting on device. These results not only verify that the output signals generated from the $\mathrm{ZnO} \mathrm{NG}$ but also signify that connecting NGs in series can increase voltage output and connecting NGs in parallel can also enhance the output current. As for practical applications, the NGs both high output and good performance in other aspects are required to better accommodate the environment and human activity. The integration of different NGs in series and parallel is of dramatic importance in the development of nano devices.

\section{Conclusion}

We have successfully grown $\mathrm{ZnO}$ nanorods on the surface of the $\mathrm{Cu}$ substrate using a simple hydrothermal method at low temperature $<100^{\circ} \mathrm{C}$. The analyses like XRD and SEM clearly indicated that the resulting $\mathrm{ZnO}$ nanorods are a wurtzite crystal structure and the crystalline rods have been grown preferentially in (002) direction, leading to the growth of onedimensional $\mathrm{ZnO}$ nanocrystals. Then, we developed a nanogenerator based on $\mathrm{ZnO}$ nanorods on $\mathrm{Cu}$ substrate which consist of twenty seven NGs in series integration to achieve enhanced output voltages of $1.8 \mathrm{~V}$ and then integrating them in parallel to obtain maximum output current of $148 \mathrm{nA}$. The simulation and experiment to check the robustness of NG were also performed. Finite element software ANSYS has been used for structural analysis. Simulated and experimental results are in close agreement. The cost effective $\mathrm{Cu}$ substrate is not only a good electrical conductor for nano devices towards real applications for effectively converting mechanical energies to electricity from the environment.

\section{Acknowledgments}

The author Naveed Sheikh is thankful to HEC Pakistan and UOB Pakistan for providing the opportunity to study at AIT Thailand through providing Ph.D. scholarship. The author Muhammad Waseem Ashraf has contributed in the simulation part of this paper only.

\section{References}

[1] R. Baron, F. W. Campbell, Streeter, L. Xiao, and R. G. Compton, "Facile method for the construction of random nanoparticle arrays on a carbon support for the development of welldefined catalytic surfaces," International Journal of Electrochemistry, vol. 3, pp. 556-565, 2008.

[2] A. B. Moghaddam, T. Nazari, J. Badraghi, and M. Kazemzad, "Synthesis of $\mathrm{ZnO}$ nanoparticles and electrodeposition of 
polypyrrole/ZnO nanocomposite film," International Journal of Electrochemical Science, vol. 4, no. 2, pp. 247-257, 2009.

[3] Z. G. Bai, D. P. Yu, H. Z. Zhang et al., "Nano-scale $\mathrm{GeO}_{2}$ wires synthesized by physical evaporation," Chemical Physics Letters, vol. 303, no. 3-4, pp. 311-314, 1999.

[4] G. Gu, M. Burghard, G. T. Kim et al., "Growth and electrical transport of germanium nanowires," Journal of Applied Physics, vol. 90, no. 11, pp. 5747-5751, 2001.

[5] Y. Cui, L. J. Lauhon, M. S. Gudiksen, J. Wang, and C. M. Lieber, "Diameter-controlled synthesis of single-crystal silicon nanowires," Applied Physics Letters, vol. 78, no. 15, pp. 22142216, 2001.

[6] M. H. Huang, Y. Wu, H. Feick, N. Tran, E. Weber, and P. Yang, "Catalytic growth of zinc oxide nanowires by vapor transport," , Advanced Materials, vol. 13, pp. 113-116, 2001.

[7] M. H. Huang, S. Mao, H. Feick et al., "Room-temperature ultraviolet nanowire nanolasers," Science, vol. 292, no. 5523, pp. 18971899, 2001.

[8] G. Shi, C. M. Mo, W. L. Cai, and L. D. Zhang, "Photoluminescence of $\mathrm{ZnO}$ nanoparticles in alumina membrane with ordered pore arrays," Solid State Communications, vol. 115, no. 5, pp. 253256, 2000.

[9] S. Xu and Z. L. Wang, "One-dimensional ZnO nanostructures: solution growth and functional properties," Nano Research, vol. 4, no. 11, pp. 1013-1098, 2011.

[10] S. Baruah and J. Dutta, "Hydrothermal growth of $\mathrm{ZnO}$ nanostructures," Science and Technology of Advanced Materials, vol. 10, no. 1, Article ID 013001, 2009.

[11] L. Vayssieres, K. Keis, S. E. Lindquist, and A. Hagfeldt, "Purpose-built anisotropie metal oxide material: 3D highly oriented microrod array of ZnO," Journal of Physical Chemistry B, vol. 105, no. 17, pp. 3350-3352, 2001.

[12] M. K. Hossain, S. C. Ghosh, Y. Boontongkong, C. Thanachayanont, and J. Dutta, "Growth of Zinc Oxide nanowires and nanobelts for gas sensing applications," Journal of Metastable and Nanocrystalline Materials, vol. 23, pp. 27-30, 2005.

[13] A. Sugunan, H. C. Warad, M. Boman, and J. Dutta, "Zinc oxide nanowires in chemical bath on seeded substrates: role of hexamine," Journal of Sol-Gel Science and Technology, vol. 39, no. 1, pp. 49-56, 2006.

[14] J. H. Lee, I. C. Leu, Y. W. Chung, and M. H. Hon, "Fabrication of ordered $\mathrm{ZnO}$ hierarchical structures controlled via surface charge in the electrophoretic deposition process," Nanotechnology, vol. 17, no. 17, pp. 4445-4450, 2006.

[15] M. Wei, D. Zhi, and J. L. MacManus-Driscoll, "Self-catalysed growth of zinc oxide nanowires," Nanotechnology, vol. 16, no. 8, pp. 1364-1368, 2005.

[16] S. H. Jo, D. Banerjee, and Z. F. Ren, "Field emission of zinc oxide nanowires grown on carbon cloth," Applied Physics Letters, vol. 85, no. 8, pp. 1407-1409, 2004.

[17] R. B. M. Cross, M. M. De Souza, and E. M. Sankara Narayanan, "A low temperature combination method for the production of ZnO nanowires," Nanotechnology, vol. 16, no. 10, pp. 2188-2192, 2005.

[18] M. Lee, C. Y. Chen, S. Wang et al., "A hybrid piezoelectric structure for wearable nanogenerators," Advanced Materials, vol. 24, no. 13, pp. 1759-1764, 2012.

[19] Y. Yang, J. Jung, B. Yun et al., "Flexible pyroelectric nanogenerators using a composite structure of lead-free $\mathrm{KNbO}_{3}$ nanowires," Advanced Materials, vol. 24, no. 39, pp. 5357-5362, 2012.
[20] G. Zhu, Z. Lin, Q. Jing et al., "Toward large-scale energy harvesting by a nanoparticle-enhanced triboelectric nanogenerator," Nano Letters, vol. 13, no. 2, pp. 847-853, 2013.

[21] C. L. Du, Z. B. Gu, Y. M. You et al., "Resonant Raman spectroscopy of (Mn, Co)-codoped ZnO films," Journal of Applied Physics, vol. 103, no. 2, Article ID 023521, 2008.

[22] S. Lee, J. Hong, C. Xu et al., "Toward robust nanogenerator using aluminum substrate," Advanced Materials, vol. 24, no. 32, pp. 4398-4402, 2012.

[23] S. Xu, Y. Qin, C. Xu, Y. Wei, R. Yang, and Z. L. Wang, "Selfpowered nanowire devices," Nature Nanotechnology, vol. 5, no. 5, pp. 366-373, 2010.

[24] C. Huang, J. Huang, S. Lin, W. Chang, J. He, and Y. Chueh, " $\mathrm{ZnO}_{1-x}$ nanorod arrays/ $\mathrm{ZnO}$ thin film bilayer structure: from homojunction diode and high-performance memristor to complementary 1D1R application," ACS Nano, vol. 6, no. 9, pp. 84078414, 2012.

[25] M. Faisal, S. B. Khan, M. M. Rahman, A. Jamal, A. M. Asiri, and M. M. Abdullah, "Synthesis, characterizations, photocatalytic and sensing studies of $\mathrm{ZnO}$ nanocapsules," Applied Surface Science, vol. 258, no. 2, pp. 672-677, 2011.

[26] M. Faisal, S. B. Khan, M. M. Rahman, A. Jamal, and M. M. Abdullah, "Fabrication of $\mathrm{ZnO}$ nanoparticles based sensitive methanol sensor and efficient photocatalyst," Applied Surface Science, vol. 258, no. 19, 75157522 pages, 2012.

[27] L. Ding, E. Yifeng, L. Fan, and S. Yang, "Growing vertical ZnO nanorod arrays within graphite: efficient isolation of large size and high quality single-layer grapheme," Chemical Communications, vol. 49, pp. 6286-6288, 2013.

[28] G. Wrobel, M. Piech, P. Gao, and S. Dardona, "Direct synthesis of $\mathrm{ZnO}$ nanorod field emitters on metal electrodes," Crystal Growth \& Design, vol. 12, pp. 5051-5055, 2012.

[29] X. Liu, C. Wang, J. Xu et al., "Fabrication of $\mathrm{ZnO} / \mathrm{CdS} /$ $\mathrm{Cu}_{2} \mathrm{ZnSnS}_{4}$ p-n heterostructure nanorod arrays via a solutionbased route," CrystEngComm, vol. 15, pp. 1139-1145, 2013.

[30] Z. Chen, N. Zhang, and Y. Xu, "Synthesis of grapheme-ZnO nanorod nanocomposites with improved photoactivity and anti-photocorrosion," CrystEngComm, vol. 15, pp. 3022-3030, 2013.

[31] A. Umar, M. M. Rahman, M. Vaseem, and Y. B. Hahn, "Ultrasensitive cholesterol biosensor based on low-temperature grown $\mathrm{ZnO}$ nanoparticles," Electrochemistry Communications, vol. 11, no. 1, pp. 118-121, 2009.

[32] W. Hu, Z. Lu, Y. Liu, T. Chen, X. Zhou, and C. M. Li, "A portable flow-through fluorescent immunoassay labon-a-chip device using $\mathrm{ZnO}$ nanorod-decorated glass capillaries," Lab on a Chip, vol. 13, pp. 1797-1802, 2013.

[33] R. Gao, J. Tian, Z. Liang, Q. Zhang, L. Wang, and G. Cao, "Nanorod-nanosheet hierarchically structured $\mathrm{ZnO}$ crystals on zinc foil as flexible photoanodes for dye-sensitized solar cells," Nanoscale, vol. 5, pp. 1894-1901, 2013.

[34] T. Jiang, T. Xie, L. Chen, Z. Fu, and D. Wang, "Carrier concentration-dependent electron transfer in $\mathrm{Cu} 2 \mathrm{O} / \mathrm{ZnO}$ nanorod arrays and their photocatalytic performance," Nanoscale, vol. 5, pp. 2938-2944, 2013.

[35] N. Sheikh, N. Afzulpurkar, M. W. Ashraf, and S. Tayyaba, "Pyramid shape $\mathrm{ZnO}$ solid microstructures," Science Series Data Report, vol. 5, no. 7, pp. 8-14, 2013. 

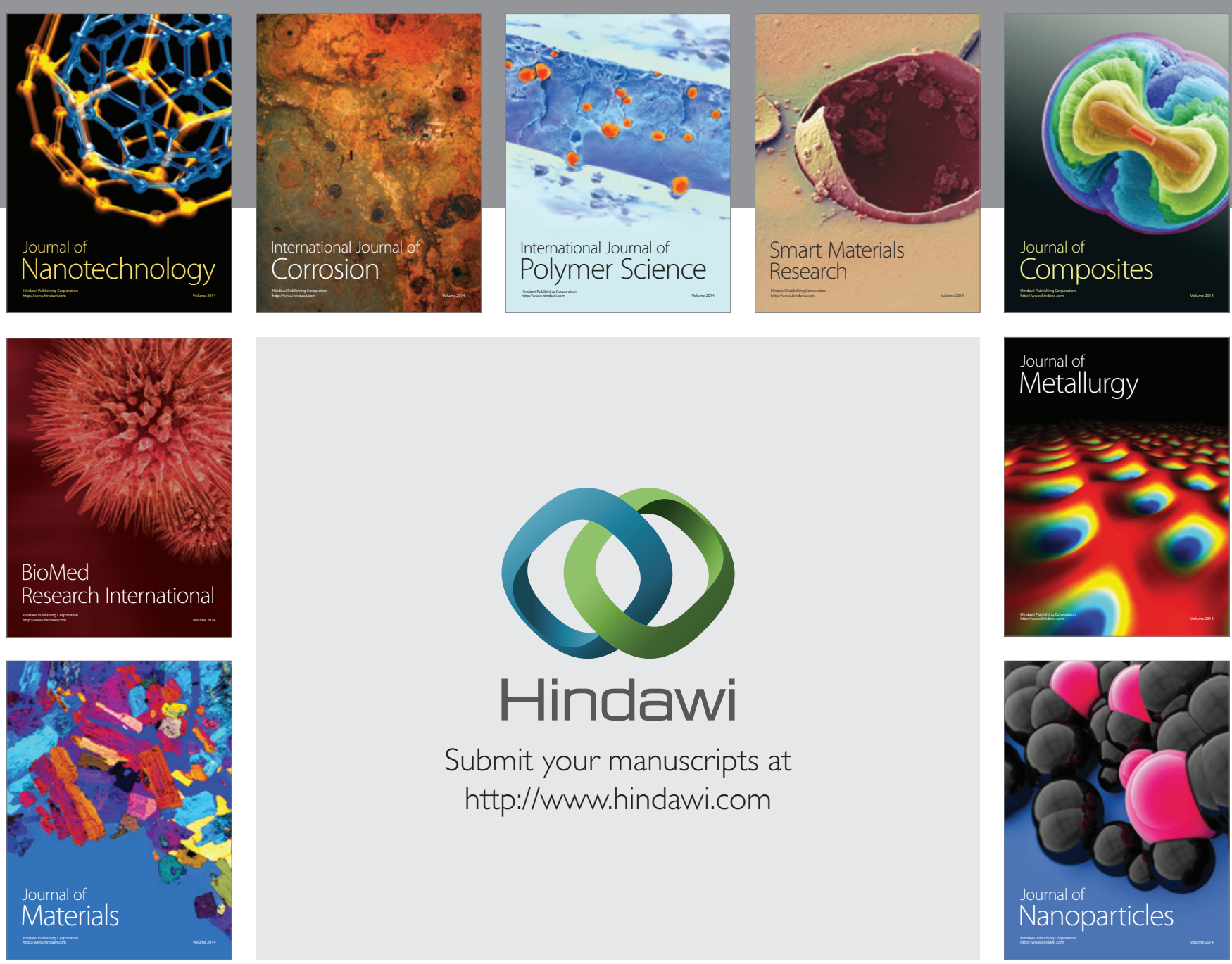

Submit your manuscripts at http://www.hindawi.com
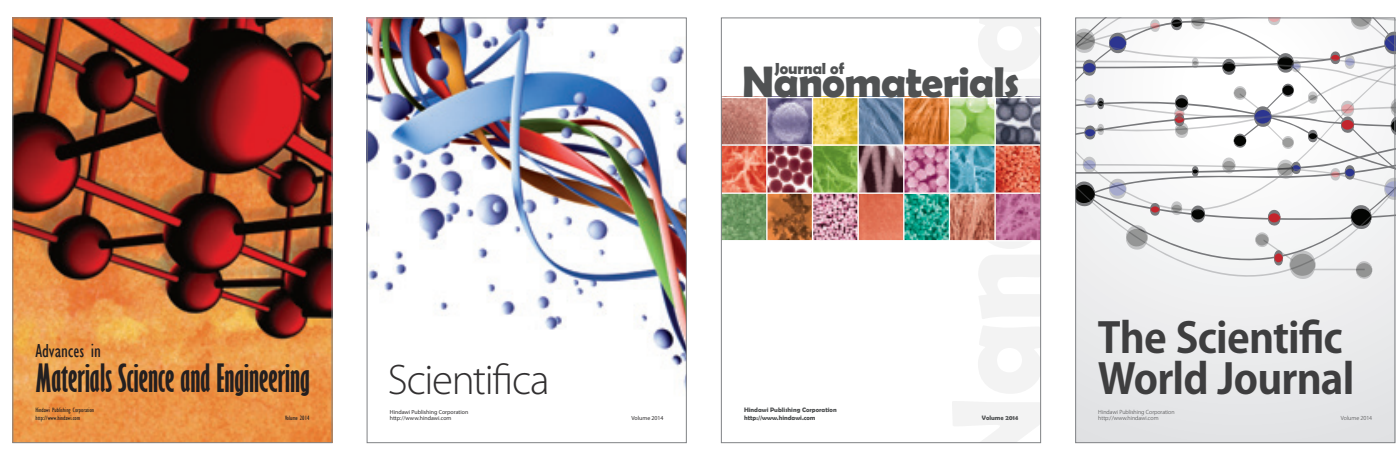

\section{The Scientific World Journal}
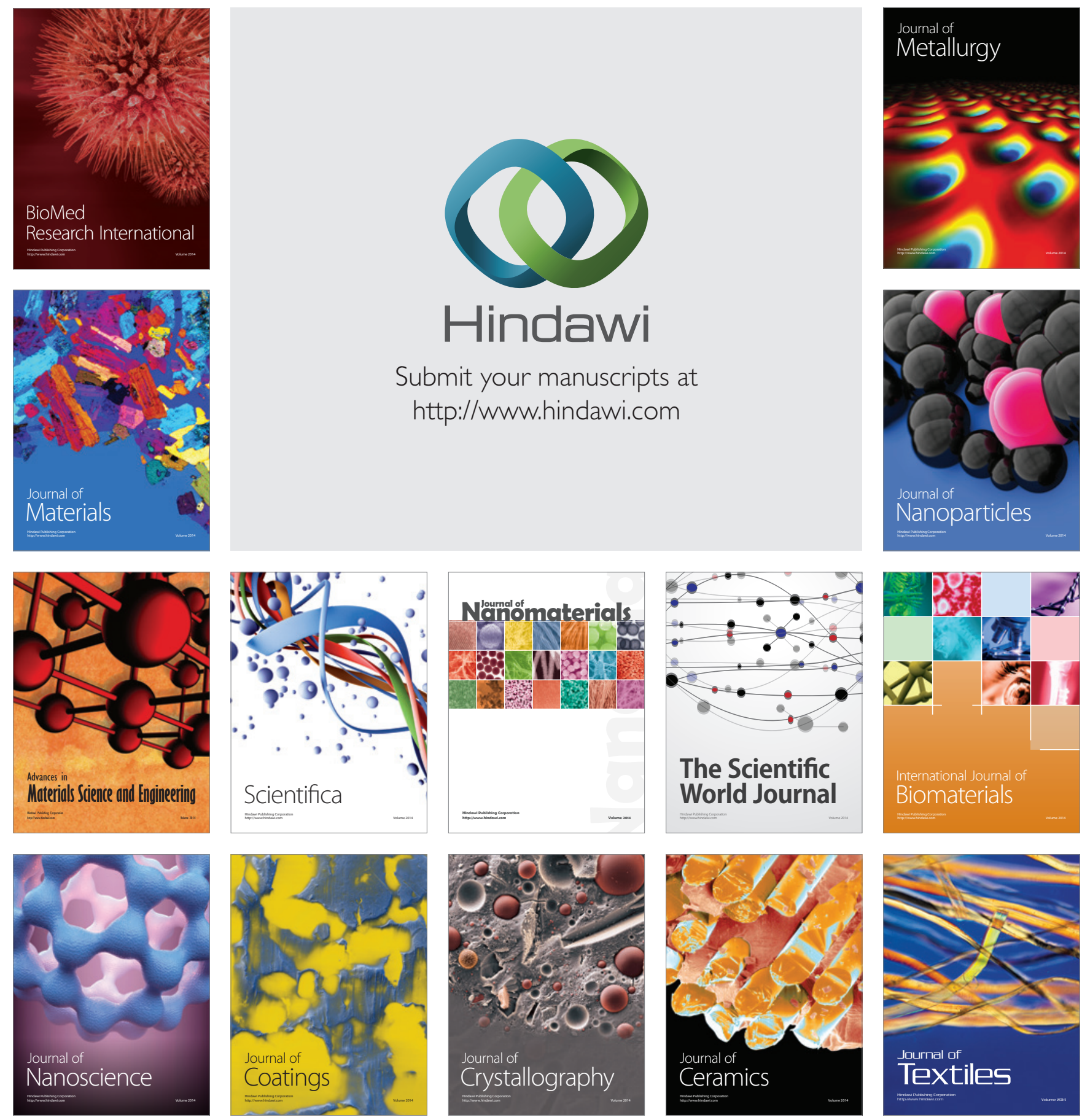